\title{
The use of Ventilator Parameters in the Prediction of Development and Outcome of Acute Respiratory Distress Syndrome in Postoperative Patients
}

\section{Chew-Ten Kor}

Internal Medicine Research Center, Department of Research, Changhua Christian Hospital, Changhua 500, Taiwan

\section{Kai-Huang Lin}

Division of Critical Care Medicine, Department of Internal Medicine, Changhua Christian Hospital, Changhua 500, Taiwan

\section{Chen-Hsu Wang}

Medical Intensive Care Unit, Division of Cardiology, Department of Internal Medicine, Cathay General Hospital, Taipei 106, Taiwan

Jui-Feng Lin

Division of Neurosurgery, Department of Surgery, Mackay Memorial Hospital, Taipei 104, Taiwan

Cheng-Deng Kuo ( $\sim$ cdkuo23@gmail.com )

Department of Medical Research, Taipei Veterans General Hospital, Taipei 112, Taiwan

\section{Research Article}

Keywords: acute respiratory distress syndrome, rapid shallow breath index, work of ventilation, rate pressure product of ventilation, inspiration to expiration time ratio

Posted Date: January 18th, 2021

DOI: https://doi.org/10.21203/rs.3.rs-145686/v1

License: (1) This work is licensed under a Creative Commons Attribution 4.0 International License. Read Full License 


\section{The use of ventilator parameters in the prediction of development and outcome of acute respiratory distress syndrome in postoperative patients}

Chew-Teng Kor, $\mathrm{PhD}^{\mathrm{a}, \mathrm{b \dagger}}$ Kai-Huang Lin, $\mathrm{MD}^{\mathrm{c} \dagger}$ Chen-Hsu Wang, MD ${ }^{\mathrm{d}}$ Jui-Feng Lin, MD ${ }^{\mathrm{e}, \mathrm{f}}$ I-Chen Chen, $\mathrm{MS}^{\mathrm{g}}$ Cheng-Deng Kuo, MD, $\mathrm{PhD}^{\mathrm{h}, \mathrm{ij}}$

a Internal Medicine Research Center, Department of Research, Changhua Christian Hospital, Changhua 500, Taiwan. Email: $179297 @$ cch.org.tw

${ }^{\mathrm{b}}$ Graduate Institute of Statistics and Information Science, National Changhua University of Education, Changhua, Taiwan

${ }^{c}$ Division of Critical Care Medicine, Department of Internal Medicine, Changhua Christian Hospital, Changhua 500, Taiwan. Email: 54407@ cch.org.tw

${ }^{\mathrm{d}}$ Medical Intensive Care Unit, Division of Cardiology, Department of Internal Medicine, Cathay General Hospital, Taipei 106, Taiwan

${ }^{\text {e }}$ Division of Neurosurgery, Department of Surgery, Mackay Memorial Hospital, Taipei 104, Taiwan

${ }^{\mathrm{f}}$ Institute of Traditional Medicine, National Yang-Ming University School of Medicine, Taipei 112, Taiwan 
${ }^{\mathrm{g}}$ Intensive Care Unit, National Taiwan University Hospital, Taipei 100, Taiwan

${ }^{\mathrm{h}}$ Division of Chest Medicine, Department of Internal Medicine, Changhua Christian Hospital, Changhua 500, Taiwan

${ }^{i}$ Department of Medical Research, Taipei Veterans General Hospital, Taipei 112, Taiwan

${ }^{j}$ Tanyu Research Laboratory, Taipei 112, Taiwan

Author contribution: Dr. Chew-Teng Kor and Dr. Kai-Huang Lin contributed equally to this study.

Corresponding author and address for reprints: Cheng-Deng Kuo, MD, PhD, Prof.

Department of Medical Research, Taipei Veterans General Hospital, Taipei 112, Taiwan. Phone: +8864 7238595 ext. 2675; E-mail: cdkuo23@gmail.com 


\section{Abstract:}

Background: To investigate the usefulness of ventilator parameters in the prediction of development and outcome of acute respiratory distress syndrome (ARDS) in postoperative patients with esophageal or lung cancer on admission to the surgical intensive care unit (SICU). Methods: A total of 32 post-operative patients with lung or esophageal cancer from SICU in a tertiary medical center were retrospectively analyzed. The study patients was divided into ARDS group $(\mathrm{n}=21)$ and non-ARDS group $(\mathrm{n}=11)$. ARDS group were the post-operative patients who developed ARDS after lung or esophageal cancer surgery. The ventilator variables were analyzed in this study. Principal component analysis (PCA) was performed to reduce the correlated ventilator variables to a small set of variables. By using the PCA selection method, top three ventilator variables with large coefficients can be considered as sensitive variables and were included in the analysis model based on the rule of 10 events per variable. Firth logistic regression with selective stepwise elimination procedure was performed to identify the most important predictors of morbidity and mortality in patients with ARDS. The ventilator parameters including rapid shallow breath index during mechanical ventilation (RSBIv), rate pressure product of ventilation (RPPv), rate pressure volume index (RPVI), mechanical work (MW), and inspiration to expiration time ratio (IER) were analyzed in this study

Results: The newly defined parameter MW/IER was the most important predictors for the 
development of ARDS, and both RPPv and RPVI were the significant predictors of mortality in patients with ARDS.

Conclusion: Some ventilator parameters can be derived from ventilator readings and be used to predict the development and outcome of ARDS in mechanically ventilated patients on admission to the SICU, such as RPPv, RPVI and MW/IER defined in this study.

Keywords: acute respiratory distress syndrome; rapid shallow breath index; work of ventilation; rate pressure product of ventilation; inspiration to expiration time ratio 


\section{Background}

Acute respiratory distress syndrome (ARDS), a life threatening inflammatory lung disease that affects both medical and surgical patients, was first reported by Ashbaugh and colleagues in 1967

in a case series of 12 ICU patients. ${ }^{1}$ The clinical, radiological, biochemical and pathological features of ARDS was defined in 1994 by the American-European Consensus Conference (AECC). ${ }^{2}$ The current definition of ARDS is the "Berlin definition" devised by a panel of experts based on the timing of clinical insult, radiographic pattern, ratio of partial pressure of arterial oxygen to fraction of inspired oxygen $\left(\mathrm{PaO}_{2} / \mathrm{FiO}_{2}\right)$ and positive end-expiratory pressure (PEEP) in 2013. ${ }^{3}$ ARDS is associated with an extremely high mortality rate in patients with lung resection or esophagectomy. ${ }^{4-8}$

ARDS is a diffuse, progressive inflammatory lung disease with hypoxemia, reduced lung compliance, bilateral opacities on chest x-ray image. Although mechanical ventilation provides essential life support, it can also induce and worsen the lung injury. ${ }^{9-10}$ However, the mechanical ventilation strategies till now remain the mainstay of respiratory support for patients with ARDS. Due to the progress of modern digital technology, advanced new-generation ventilators allow extensive and integrated monitoring of patients' respiratory mechanics. Data regarding the control variables, phase variables, breath type, respiration rates, tidal volumes and so on, can be easily read and recorded constantly from the ventilator. 
In this study we investigated the usefulness of ventilator parameters derived from the ventilator readings in the prediction of development and outcome of ARDS in postoperative patients with lung or esophageal cancer on admission to the surgical ICU (SICU).

\section{Methods}

\section{Study Cohort and Design}

This was a prospective, single-centric, case-controlled study with retrospective data analysis. The study protocol has been approved by Institute Review Board of National Taiwan University Hospital (NTUH200808065R) and Taipei Veterans General Hospital (VGHIRB97-01-02A). Retrospective analysis of the data to find the key ventilator parameters for the prediction of development and outcome of ARDS in postoperative patients with esophageal or lung cancer has been approved by the Institute Review Board of Changhua Christian Hospital (CCH IRB 190521).

This study was conducted in the SICU of the National Taiwan University Hospital. All patients were over 18 years old, and had received thoracic surgery for lung or esophageal cancer. They were transferred to the SICU for post-operative care. Patients without post-operative ARDS were included as the control group, while patients complicated with ARDS were included as the ARDS group. The ARDS of the patients was diagnosed according to the Berlin Definition. Patients who had severe coronary artery disease, persistent arrhythmia, cardiac pacing, diabetes mellitus, 
cerebral vascular accident, or major diseases of kidney or autoimmune system were excluded from the study. Patient selection criteria in this study have been presented in our previous article. ${ }^{[1]}$ Eleven patients in the non-ARDS group and 21 patients in the ARDS group were analyzed in this study.

All patients were intubated and mechanically ventilated using pressure control mode. Fentanyl was given to all patients as an analgesic. The demographic data, vital signs, medications, ventilator readings, and relevant clinical data were recorded within 4 hours of admission to the SICU.

\section{Ventilator Parameters}

Flow rate $(\dot{V})$ during mechanical ventilation is given by the following equation:

$$
\dot{\mathrm{V}}=\frac{\mathrm{V}_{\mathrm{T}}}{\mathrm{Ti}},
$$

where $\mathrm{V}_{\mathrm{T}}$ is the tidal volume and $\mathrm{Ti}$ is the inspiration time. Pressure control mode of ventilation typically has decelerating pattern and $\mathrm{V}_{\mathrm{T}} / \mathrm{Ti}$ will provide average flow rate which depends on both resistance and compliance. The flow resistance $(\mathrm{R})$ of air during mechanical ventilation is given by the ratio of peak inspiratory pressure above PEEP (PIP) to the flow rate $\dot{V}$

$$
\mathrm{R}=\frac{\mathrm{PIP}}{\dot{\mathrm{V}}} .
$$

The dynamic compliance (Cdyn) represents the pulmonary compliance during inspiration, and is given by the ratio of tidal volume to the PIP during inspiration 


$$
\text { Cdyn }=\frac{\mathrm{V}_{\mathrm{T}}}{\mathrm{PIP}}
$$

The rapid shallow breathing index (RSBI) is thought to be a stress response reflecting the balance between respiratory neuromuscular reserve and respiratory demands, and has been widely used in daily clinical practice such as the prediction of successful weaning from ventilator. ${ }^{[12-15]}$ When the patient is on the ventilator using pressure control or pressure support mode of ventilation, the RSBI during mechanical ventilation (RSBIv) can also be defined as the ratio of respiration rate (RR) to the tidal volume

$$
\text { RSBIV }=\frac{\mathrm{RR}}{\mathrm{V}_{\mathrm{T}}}
$$

The subscript ' $v$ ' in RSBIv indicates that this RSBIv is measured when the patient is still on the ventilator, which is different from the RSBI measured when the patient is temporarily discontinued from mechanical ventilation.

The product of heart rate (HR) and systolic blood pressure (SBP), or the rate-pressure product (RPP), is a very reliable indicator of myocardial oxygen demand, and has been widely used clinically, especially in cardiology, anesthesiology and rehabilitation. ${ }^{16-18}$ By analogy with the RPP in cardiology, the rate pressure product of ventilation (RPPv) can be defined as the product of RR and PIP in ventilated patients to measure the stress imposed on the respiratory muscle in ventilated patients:

$$
\mathrm{RPPV}=\mathrm{RR} \cdot \mathrm{PIP}
$$


Combining RSBIv and RPPv, we can devise the rate pressure volume index (RPVI) as

$$
\mathrm{RPVI}=\mathrm{RSBIV} \cdot \mathrm{PIP}=\frac{\mathrm{RR} \cdot \mathrm{PIP}}{\mathrm{V}_{\mathrm{T}}}=\frac{\mathrm{RPPV}}{\mathrm{V}_{\mathrm{T}}}
$$

Though RR appears in both RSBIv and RPPv, it is included in the definition of RPVI only once rather than twice so as not to overemphasize its role in the new index of RPVI.

The respiratory interval (RI) is the averaged time of ventilation that can be obtained from the RR using the following equation

$$
\mathrm{RI}=\frac{60 \mathrm{sec}}{\mathrm{RR}}
$$

The expiration time (Te) is the difference between RI and $\mathrm{Ti}$

$$
\mathrm{Te}=\mathrm{RI}-\mathrm{Ti}
$$

The inspiration to expiration time ratio (IER) is then

$$
\mathrm{IER}=\frac{\mathrm{Ti}}{\mathrm{Te}}
$$

When the gas flows across a constant cross section, the mechanical work (MW) done by the ventilator can be defined as

$$
\mathrm{MW}=\mathrm{PIP} \cdot \mathrm{V}_{\mathrm{T}}
$$

which is different from the conventional work of breathing work done by the patient when the patient is breathing on his/her own effort. Since MW is related to both inspiration and expiration times, a new parameter MW/IER can be constructed to reflect the severity of ARDS

$$
\frac{M W}{\mathrm{IER}}=\left(\mathrm{PIP} \cdot \mathrm{V}_{\mathrm{T}}\right) \frac{\mathrm{Te}}{\mathrm{Ti}}=\mathrm{PIP} \cdot \dot{\mathrm{V}} \cdot \mathrm{Te}
$$




\section{Statistical Analysis}

Data are presented as a percentage, median (interquartile range) or mean \pm standard deviation.

Continuous variables were tested for normal distribution using the Kolmogorov-Smirnov test, and were compared between the two groups of patients using Mann-Whitney $U$ test for non-normally distributed data, or independent samples $t$-test for normally distributed data. Chi-square test or Fisher's exact test, when appropriate, was used for the comparisons of categorical data.

Firth logistic regression with selective stepwise elimination procedure was performed to identify the most important predictors of morbidity and mortality of ARDS. This method used the penalty likelihood approach to reduce the parameter estimation bias due to small sample size. However, only three variables can be included in the multivariable analysis based on the rule of 10 events per variable (EPV-10), as in our previous study. ${ }^{11}$ The candidate of mechanical ventilation measurements, included in the multivariable analysis, were the significant variables in the univariate analysis of differences between the two groups. Since these mechanical ventilation measurements were highly correlated with one another, principal component analysis (PCA) was performed to reduce the correlated variables to a small set of variables. By using the PCA selection method, variables with large coefficients in each component can be considered as sensitive variables because those variables contain most information from the dataset. In this study, the top three variables were selected as the sensitive variables. Furthermore, receiver-operating 
characteristic (ROC) curves were constructed to assess the predictive performance of important

measurements. All statistical analyses were performed using R software (version i386 3.3.2, https://www.r-project.org/) and the contributed R package logistic for Firth's penalized-likelihood logistic regression. A two-tailed $p<0.05$ was considered statistically significant.

\section{Results}

Table 1 shows that the ARDS patients had significantly longer SICU stay, greater RR, R, RSBIv, RPPv, RPVI, PEEP, and IER, and significantly smaller RI, Te, Vं, V than the control patients. Table 2 shows that the non-survivors had significantly greater PIP, RSBIv, RPPv, and RPVI than the survivors of ARDS.

Two components were extracted after PCA procedure and the coefficients of variables in each component were summarized in Table 3. The variables in the same column of shaded area in Table 3 indicate that they are included in the same component. By using PCA, the RSBIv, RPVI, MW/IER were found to be the sensitive variables in component 1 , and the $\dot{V}$, MW and $\mathrm{V}_{\mathrm{T}}$ were found to be the sensitive variables in component 2 . Three models were used to assess the predictors of morbidity of ARDS in multivariable analysis. Model 1a and model 2a were adjusted for three sensitive variables in components 1 and 2, respectively. After performing selective stepwise elimination procedure for model 1a and model 2a, both MW/IER $(\mathrm{p}=0.006)$ and $\mathrm{V}_{\mathrm{T}}(\mathrm{p}=0.033)$ 
were shown to be the significant predictors for the development of ARDS (Table 4). Model 3a adjusted for MW/IER and $\mathrm{V}_{\mathrm{T}}$, and showed that the MW/IER was the most important predictor for the development of ARDS (Table 4). As depicted in Figure 1A, the AUCs and 95\% confidence intervals (CI) of PEEP, RSBIv, RPVI, $\mathrm{V}_{\mathrm{T}}$ and $\dot{\mathrm{V}}$ in predicting the morbidity of ARDS were 0.931 $(95 \% \mathrm{CI}=0.85-1.00 ; p<0.001), 0.885(95 \% \mathrm{CI}=0.77-1.00 ; p<0.001), 0.857(95 \% \mathrm{CI}=0.77$ $-1.00 ; p<0.001), 0.816(95 \% \mathrm{CI}=0.67-1.00 ; p=0.004)$ and $0.801(95 \% \mathrm{CI}=0.65-1.00 ; p=$ 0.006), respectively. The AUC of the new parameter MW/IER was found to be $0.944(95 \% \mathrm{CI}=$ $0.86-1.00 ; p<0.001)$

The PIP, RSBIv, RPPv, and RPVI were the significant variables responsible for the differences between the survivors and non-survivors of ARDS (Table 2). Since the RSBIv, RPPv, and RPVI are highly correlated with one another, we used three separate model to assess the predictors of mortality of ARDS. Model $1 \mathrm{~b}$ adjusted for PIP and RSBIv, and indicated that none of the parameters in model $1 \mathrm{~b}$ could significantly affect the mortality of ARDS $(p>0.10)$. Model $2 \mathrm{~b}$ and model $3 \mathrm{~b}$ adjusted for RPPv and RPVI, respectively. The results of RPPV and RPVI could marginally significantly affect the mortality of ARDS $(p<0.10)$ (Table 4). As depicted in Figure 1B, the AUCs of RPVI, RPPv, RSBI and PIP in predicting the mortality of ARDS were 0.850 $(95 \% \mathrm{CI}=0.68-1.00 ; p=0.021), 0.838(95 \% \mathrm{CI}=0.66-1.00 ; p=0.026), 0.706(95 \% \mathrm{CI}=0.49-$ $1.00 ; p=0.173)$ and $0.813(95 \% \mathrm{CI}=0.63-1.00 ; p=0.039)$, respectively. 


\section{Discussion}

Despite significant advances in the understanding and treatment of ARDS in the past fifty years, the mortality rate of ARDS remains high. ${ }^{19}$ The risk factors and outcome evaluation of ARDS are still important issues for mortality reduction. Many indices have been developed to predict the risk and outcomes of postoperative ARDS in trauma or surgical patients. ${ }^{6,820-23}$ However, these indices or scoring system require the inclusion of a broad range of risk factors and risk modifiers that are challenging in clinical practice. It is necessary to find some simple and powerful parameters or scores to predict the development and to evaluate the risk of ARDS.

This study investigated the usefulness of ventilator parameters in the prediction of development and outcome of ARDS in mechanically ventilated patients on admission to the SICU.

We found that a small value of MW/IER was the most important predictor for the development of ARDS, and the large values of RPPv and RPVI were marginally significant predictors of mortality of ARDS. These results suggest that ventilator parameters can be used to predict the morbidity and mortality of ARDS in postoperative patients with esophageal or lung cancer on admission to the SICU.

The RSBI is thought to be a stress response reflecting the balance between respiratory neuromuscular reserve and respiratory demands. It is a clinical parameter often used in the prediction of successful weaning from ventilators in ventilated patients. ${ }^{12-15}$ The RSBI is obtained 
by measuring the respiratory rate and tidal volume of the patient without ventilatory support, while the RSBIv defined in this study was measured when the patient was still on the ventilator. Thus, the RSBIv is different from the RSBI used clinically to predict weaning outcome of the patients, though the equations used to calculate the RSBI and RSBIv are the same. Since the lungs of the ARDS patients were more rigid than the controls, their tidal volumes were smaller and their respiratory rates was greater, resulting in a greater RSBIv in ARDS patients, as compared to the control patients. That the RSBIv of non-survived ARDS patients was greater than that of survived ARDS patients can also be accounted for by the more rigid lung in the non-survived ARDS patients. Although the mean value of RSBIv was significantly higher in ARDS patients as well as in nonsurvived ARDS patients, the increase in RSBIv did not increase the risk of development of ARDS and the mortality risk of ARDS patients in adjustment model. Though the RSBIv might be used to indicate the severity of lung involvement, it cannot be used to predict the development of ARDS and the mortality of ARDS in mechanically ventilated patients on admission to the SICU.

The MW of the ARDS patients was found to be smaller than that of the control patients in this study. This should not be interpreted as the lesser respiratory distress in the ARDS patients as compared to the control patients, because the MW measured the support delivered to the patients by the ventilators which was not the same as the work of breathing measured in patients who are breathing on their own efforts. The smaller MW in ARDS patients compared to control patients 
might be caused mainly by the smaller $\mathrm{V}_{\mathrm{T}}$ due to edematous lungs in such patients. A small $\mathrm{V}_{\mathrm{T}}$ can also lead to a smaller Te, resulting in a greater IER in ARDS patients as compared to the control patients.

By analogy with the RPP in cardiology, we define the RPPv to measure the stress imposed on the respiratory muscle in ventilated patients. In addition, by combining the RSBIV with RPPv, we can define the RPVI in ventilated patients. We found that both RPPv and RPVI of the ARDS patients were greater than those of the control patients. In ARDS patients, the greater RPPv was caused by the greater RR, and the greater RPVI was caused by the greater RR and the smaller $\mathrm{V}_{\mathrm{T}}$. Moreover, we found that both RPPv and RPVI of the non-survived ARDS patients were greater than those of the survived ARDS patients. The greater RPVI in the non-survived ARDS was caused by a greater PIP, and the greater RPVI in the same subgroup of patients was caused by a greater PIP and a smaller $\mathrm{V}_{\mathrm{T}}$ in those ARDS patients. In adjustment model, both RPPv and RPVI were marginally significant predictors for the mortality of ARDS patients. This result suggested that both RPPv and RPVI might have prognostic value in the management of ARDS patients.

The major limitation of this study was the small number of ARDS patients. The reason have been presented in our previous article. ${ }^{11}$ In order to resolve this issue, the PCA selection method, EPV-10 principle and Firth logistic regression of penalty likelihood approach were chosen in our investigation so that our findings could be substantiated. 


\section{Conclusions}

Some ventilator parameters can be defined and used to predict the development and outcome of the mechanically ventilated ARDS patients on admission to the SICU, such as RPPv, RPVI and MW/IER defined in this study. A larger scale study is needed to verify this finding and identify the most useful ventilator parameters in the prediction of development and outcome of ARDS patients.

\section{Abbreviations}

ARDS: acute respiratory distress syndrome; SICU: surgical intensive care unit; RSBIv: rapid shallow breath index during mechanical ventilation; RPPv: rate pressure product of ventilation; RPVI: rate pressure volume index; MW: mechanical work; IER: inspiration to expiration time ratio; $\mathrm{PaO} 2$ : partial pressure of arterial oxygen; FiO2: fraction of inspired oxygen; PEEP: positive

end-expiratory pressure; $\dot{V}$ :Flow rate; VT: Tidal volume; Ti: inspiration time; R: flow resistance; PIP: ratio of peak inspiratory pressure above PEEP; Cdyn: The dynamic compliance; RSBIv: The rapid shallow breathing index RSBI during mechanical ventilation; RR: ratio of respiration rate; HR: The product of heart rate; SBP: systolic blood pressure; RPP: the rate-pressure product; RPPv: the rate pressure product of ventilation; RPVI: the rate pressure volume index; RI: the respiratory interval; Te: the expiration time; PCA: Principal component analysis; EPV-10: the 
rule of 10 events per variable; ROC: receiver-operating characteristic curves; CI: confidence intervals.

\section{Ethics approval and consent to participate}

The study protocol has been approved by the Institutional Review Boards of National Taiwan University Hospital (NTUH200808065R) and Taipei Veterans General Hospital (VGHIRB97-0102A), and written informed consent was obtained from the next of kin of the patients before their enrollment in the study.

\section{Consent for publication}

All included patients or their family members signed the informed consent form to report individual patient data. All authors have confirmed the manuscript and approved the publication of the manuscript.

\section{Availability of data and materials}

The datasets used and/or analyzed during the current study available from the corresponding author on request.

\section{Competing interests}

The authors declare that they have no competing interest.

\section{Funding}

This study was supported by the grant VN99-14 from the Taipei Veterans General Hospital- 
National Taiwan University Hospital Joint Research Program, Taipei, Taiwan, and the grant 109CCH-IRP-032 from the Changhua Christian Hospital, Changhua, Taiwan.

\section{Authors' contributions}

Conception and design of study: CDK; Acquisition of data: ICC; Analysis and/or interpretation of data: CTK, KHL; Drafting of the manuscript: CDK, KHL, CTK; Revising the manuscript critically for important intellectual content: CHW, JFL; Approval of the version of the manuscript to be published: KHL, CTK, CHW, JFL, ICC, CDK.

\section{Acknowledgements:}

Not applicable. 


\section{References}

1. Ashbaugh D, Bigelow DB, Petty T, Levine B. Acute respiratory distress in adults. Lancet 1967;2(7511):319-323.

2. Bernard GR, Artigas A, Brigham KL, Carlet J, Falke K, Hudson L, et al. The AmericanEuropean consensus conference on ARDS. Definitions, mechanisms, relevant outcomes, and clinical trial coordination. Am J Resp Crit Care Med 1994;149(3 Pt 1):818-824.

3. Ferguson ND, Fan E, Camporota L, Antonelli M, Anzueto A, Beale R, et al. The Berlin definition of ARDS: An expanded rationale, justification, and supplementary material. Intens Care Med 2012;38:1731-1732.

4. D'Annoville T, D'Journo XB, Trousse D, Brioude G, Dahan D, Seitz JF, et al. Respiratory complications after oesophagectomy for cancer do not affect disease-free survival. Eur J Cardiothorac Surg 2012;41:e66-73.

5. Dulu A, Pastores SM, Park B, Riedel E, Rusch V, Halpern NA. Prevalence and mortality of acute lung injury and ARDS after lung resection. Chest 2006;130:73-78.

6. Kim JB, Lee SW, Park SI, Kim YH, Kim DK. Risk factor analysis for postoperative acute respiratory distress syndrome and early mortality after pneumonectomy: The predictive value of preoperative lung perfusion distribution. J Thorac Cardiovasc Surg 2010;140:26-31.

7. Kometani T, Okamoto T, Yoshida S, Yoshino I. Acute respiratory distress syndrome after 
pulmonary resection. Gen Thorac Cardiovasc Surg 2013;61:504-512.

8. Tandon S, Batchelor A, Bullock R, Gascoigne A, Griffin M, Hayes N, et al. Peri-operative risk factors for acute lung injury after elective oesophagectomy. Br J Anaesth 2001;86:633638.

9. Ricard JD, Dreyfuss D, Saumon G. Ventilator-induced lung injury. Eur Resp J Suppl $2003 ; 42: 2 s-9 s$.

10. Slutsky AS, Ranieri VM. Ventilator-induced lung injury. N Eng J Med 2013;369:2126-2136.

11. Chen IC, Kor CT, Lin CH, Kuo J, Tsai JZ, Ko WJ, et al. High-frequency power of heart rate variability can predict the outcome of thoracic surgical patients with acute respiratory distress syndrome on admission to the intensive care unit: a prospective, single-centric, casecontrolled study. BMC Anesthesiol 2018;18:34.

12. Kuo PH, Kuo SH, Yang PC, Wu HD, Lu BY, Chen MT. Predictive value of rapid shallow breathing index measured at initiation and termination of a 2-hour spontaneous breathing trial for weaning outcome in ICU patients. J Formos Med Assoc 2006;105:390-398.

13. Nemer SN, Barbas CS, Caldeira JB, Cárias TC, Santos RG, Almeida LC, et al. A new integrative weaning index of discontinuation from mechanical ventilation. Crit Care 2009; 13:R152.

14. Verceles AC, Diaz-Abad M, Geiger-Brown J, Scharf SM. Testing the prognostic value of the 
rapid shallow breathing index in predicting successful weaning in patients requiring prolonged mechanical ventilation. Heart Lung 2012;41:546-552.

15. dos Santos Bien U, Souza GF, Campos ES, De Carvalho EF, Fernandes MG, Santoro I, et al. Maximum inspiratory pressure and rapid shallow breathing index as predictors of successful ventilator weaning. J Phys Ther Sci 2015;27:3723-3727.

16. Zargar JA, Naqash IA, Gurcoo SA. Comparative evaluation of the effect of metoprolol and esmolol on rate pressure product and ECG changes during laryngoscopy and endotracheal intubation in controlled hypertensive patients. Indian J Anaesth 2002;46:363-368.

17. May GA, Nagle FJ. Changes in rate-pressure product with physical training of individuals with coronary artery disease. Phys Ther 1984;64:1361-1316.

18. Forjaz CLDM, Matsudaira Y, Rodrigues FB, Nunes N, Negrão CE. Post-exercise changes in blood pressure, heart rate and rate pressure product at different exercise intensities in normotensive humans. Braz J Med Biol Res 1998;31:1247-1255.

19. Máca J, Jor O, Holub M, Sklienka P, Burša F, Burda M, et al. Past and present ARDS mortality rates: A systematic review. Respir Care 2017;62:113-122.

20. Afshar M, Smith GS, Cooper RS, Murthi S, Netzer G. Trauma indices for prediction of acute respiratory distress syndrome. J Surg Res 2016;201:394-401.

21. Becher RD, Colonna AL, Enniss TM, Weaver AA, Crane DK, Martin RS, et al. An innovative 
approach to predict the development of adult respiratory distress syndrome in patients with blunt trauma. J Trauma Acute Care Surg 2012;73:1229-1235.

22. Johnson RG, Arozullah AM, Neumayer L, Henderson WG, Hosokawa P, Khuri SF. Multivariable predictors of postoperative respiratory failure after general and vascular surgery: Results from the patient safety in surgery study. J Am Coll Surg 2007;204:1188-1198.

23. Levitt JE, Calfee CS, Goldstein BA, Vojnik R, Matthay MA. Early acute lung injury: Criteria for identifying lung injury prior to the need for positive pressure ventilation. Crit Care Med 2013;41:1929-1937. 
Table 1. Comparisons between control and ARDS groups

Table 2. Comparisons between survivors and non-survivors of patients with ARDS

Table 3. The areas under the ROC curves (AUC) of mechanical ventilator parameters and variable coefficient in principal components analysis for the development of ARDS

Table 4. Firth logistic regression analyses with stepwise elimination procedure for

predicting the development of ARDS and mortality in patients with ARDS

\section{Figure Legends}

Figure 1. The areas under the ROC curves (AUC) and 95\% confidence intervals (CI) of mechanical ventilation parameters in predicting the development of ARDS and the mortality in patients. (A) the AUCs and 95\% confidence intervals (CI) of PEEP, RSBIv,

RPVI, $\mathrm{V}_{\mathrm{T}}$ and $\dot{\mathrm{V}}$ in predicting the morbidity of ARDS were $0.931,0.885,0.857,0.816$ and 0.801, respectively. (B) the AUCs of RPVI, RPPv, RSBI and PIP in predicting the mortality of ARDS were $0.850,0.838,0.706$ and 0.813 , respectively. 

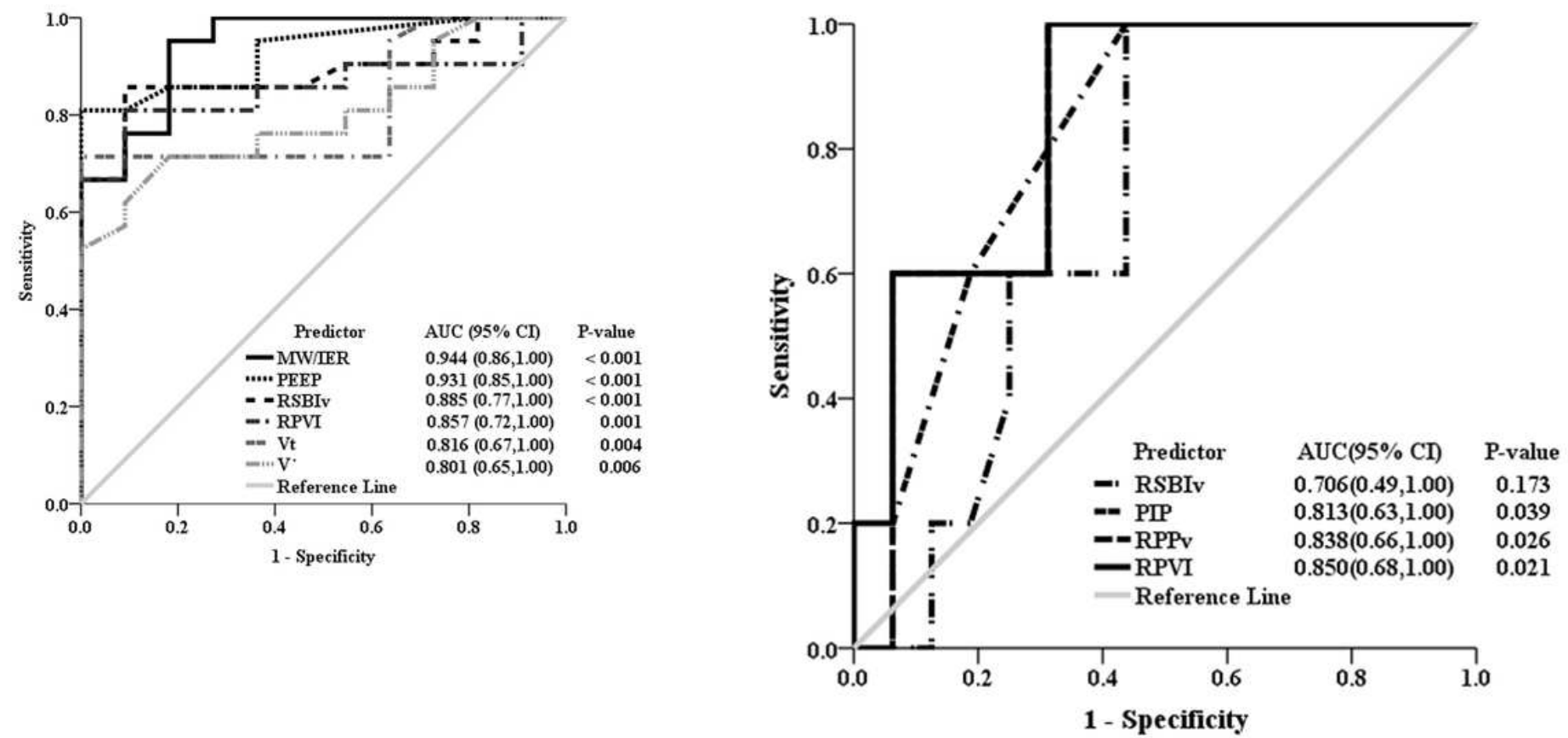

\section{Figure 1}

The areas under the ROC curves (AUC) and 95\% confidence intervals (CI) of mechanical ventilation parameters in predicting the development of ARDS and the mortality in patients. (A) the AUCs and 95\% confidence intervals $(\mathrm{Cl})$ of PEEP, RSBIv, RPVI, VT and $\mathrm{V} \otimes$ in predicting the morbidity of ARDS were 0.931 , $0.885,0.857,0.816$ and 0.801 , respectively. (B) the AUCs of RPVI, RPPv, RSBI and PIP in predicting the mortality of ARDS were $0.850,0.838,0.706$ and 0.813 , respectively.

\section{Supplementary Files}

This is a list of supplementary files associated with this preprint. Click to download.

- BMCAnesthesiologyVentinARDSTable.docx 